\title{
Asymptotic Almost Periodic Functions with Range in a Topological Vector Space
}

\author{
Liaqat Ali Khan and Saud M. Alsulami \\ Department of Mathematics, King Abdulaziz University, P.O. Box 80203, Jeddah 21589, Saudi Arabia \\ Correspondence should be addressed to Liaqat Ali Khan; akliaqat@gmail.com
}

Received 30 May 2013; Accepted 13 September 2013

Academic Editor: Sivaram K. Narayan

Copyright ( 2013 L. A. Khan and S. M. Alsulami. This is an open access article distributed under the Creative Commons Attribution License, which permits unrestricted use, distribution, and reproduction in any medium, provided the original work is properly cited.

\begin{abstract}
The notion of asymptotic almost periodicity was first introduced by Fréchet in 1941 in the case of finite dimensional range spaces. Later, its extension to the case of Banach range spaces and locally convex range spaces has been considered by several authors. In this paper, we have generalized the concept of asymptotic almost periodicity to the case where the range space is a general topological vector space, not necessarily locally convex. Our results thus widen the scope of applications of asymptotic almost periodicity.
\end{abstract}

\section{Introduction}

The theory of almost periodic functions was mainly created and published during 1924-1926 by the Danish mathematician Harold Bohr. In 1933, Bochner [1] published an important article devoted to extension of the theory of almost periodic functions on the real line $\mathbb{R}$ with values in a Banach space $E$. His results were further developed by several mathematicians, see, for example [2-7].

The concept of asymptotic almost periodicity was first considered by Fréchet $[8,9]$ in 1941 for functions $f: \mathbb{R}^{+} \rightarrow E$ with the range $E$ restricted to a finite dimensional space. The semigroup case of $\mathbb{R}^{+}$turns out to be significantly different from the group case of $\mathbb{R}$. If $E$ is a Banach space or a locally convex case and $\mathbb{R}^{+}=[0, \infty)$ replaced by $J_{a}=[a, \infty)$, with $a \in \mathbb{R}$, this notion has been extensively studied in recent years (see [10-14]). In this paper, we generalize the concept of asymptotic almost periodicity to the case of $E$, a general topological vector space.

\section{Preliminaries}

In this section, we give prerequisites on topological vector spaces and almost periodic functions for our main results of Section 3 .
Throughout this paper, $E$ denotes a nontrivial Hausdorff topological vector space (in short, a TVS) with a base $\mathscr{W}$ of closed balanced shrinkable neighborhoods of 0 . (A neighborhood $W$ of 0 in $E$ is called shrinkable [15] if $r \bar{W} \subseteq$ int $W$ for $0 \leq r<1$.) By [15, Theorem 4 and 5], every Hausdorff TVS has a base of shrinkable neighborhoods of 0 , and also the Minkowski functional $\rho_{W}$ of any such neighborhood $W$ is continuous and satisfies

$$
\begin{gathered}
\bar{W}=\left\{x \in E: \rho_{W}(x) \leq 1\right\}, \\
\text { int } W=\left\{x \in E: \rho_{W}(x)<1\right\} .
\end{gathered}
$$

We mention that, for any neighborhood $W$ of 0 in $E, \rho_{W}$ need not be absolutely homogeneous or subadditive; however, the following useful properties hold $[15,16]$.

(a) $\rho_{W}$ is positively homogeneous; it is absolutely homogeneous if $W$ is balanced.

(b) If $V$ is a balanced neighborhood of 0 in $E$ with $V+V \subseteq$ $W$, then

$$
\rho_{W}(x+y) \leq \rho_{V}(x)+\rho_{V}(y) \quad \forall x, y \in E .
$$

A complete metrizable TVS is called an F-space. 
Notations. Let $X$ be a completely regular Hausdorff space, and let $C(X, E)$ be the set of all continuous functions $E$-valued functions on $X$. Furthermore, let

$$
\begin{gathered}
C_{b}(X, E)=\{f \in C(X, E): f(X) \text { is bounded in } E\}, \\
C_{p c}(X, E)=\{f \in C(X, E): f(X) \text { is precompact in } E\}, \\
C_{0}(X, E)=\{f \in C(X, E): f \text { vanishes at infinity on } X\} .
\end{gathered}
$$

Clearly, $C_{p c}(X, E) \subseteq C_{b}(X, E) \subseteq C(X, E), C_{0}(X, E) \subseteq$ $C_{b}(X, E)$, and all these sets are vector spaces over $\mathbb{K}$ with the pointwise operations of addition and scalar multiplication. The uniform topology $u$ on $C_{b}(X, E)$ is defined as the linear topology which has a base of neighborhoods of 0 consisting of all sets of the form

$$
N_{b}(X, W)=\left\{f \in C_{b}(X, E): f(X) \subseteq W\right\},
$$

where $W \in \mathscr{W}$. The compact-open topology $k$ on $C(X, E)$ is defined as the linear topology which has a base of neighborhoods of 0 consisting of all sets of the form

$$
N(K, W)=\{f \in C(X, E): f(K) \subseteq W\},
$$

where $K \subseteq X$ is compact and $W \in \mathscr{W}$. Clearly, $k \leq u$ on $C_{b}(X, E)$. (For details, see [16].)

We state the following two general versions of the ArzelàAscoli theorem $[16,17]$ for reference purpose.

Theorem 1. Let $X$ be a locally compact space. A subset $\mathscr{A}$ of $C_{0}(X, E)$ is precompact if and only if the following conditions hold:

(i) $\mathscr{A}$ is equicontinuous:

(ii) $\mathscr{A}(x)=\{f(x): f \in \mathscr{A}\}$ is precompact in $E$ for each $x \in X$;

(iii) $\mathscr{A}$ vanishes at infinity on $X$; that is, given $W \in \mathscr{W}$, there exists a compact set $K \subseteq X$ such that $f(y) \in W$ for all $f \in \mathscr{A}$ and $y \in X \backslash K$;

Before stating the next result, we introduce the following notation: for any $\mathscr{A} \subseteq C_{p c}(X, E)$ and $W \in \mathscr{W}$, let

$$
\begin{aligned}
G_{x}(\mathscr{A}, W)=\{ & y \in X: f(y) \\
& \quad-f(x) \in W \forall f \in \mathscr{A}\}, \quad x \in X .
\end{aligned}
$$

Theorem 2. Let $X$ be a $k$-space. Then, for any $\mathscr{A} \subseteq C_{p c}(X, E)$, the following are equivalent:

(1) $\mathscr{A}$ is precompact;

(2) consider the following:

(i) $\mathscr{A}$ is equicontinuous;

(ii) $\mathscr{A}(x)$ is precompact in $E$ for each $x \in X$;

(iii) given $W \in \mathscr{W}$, there exists a compact set $K \subseteq X$ such that $\left\{G_{x}(\mathscr{A}, W): x \in K\right\}$ covers $X$;

(3) (i) $\mathscr{A}(X)=\{f(x): x \in X, f \in \mathscr{A}\}$ is precompact in E; (ii) given $\mathrm{W} \in \mathscr{W}$, there exists a finite set $\mathrm{F} \subseteq \mathrm{X}$ such that $\left\{\mathrm{G}_{\mathrm{x}}(\mathscr{A}, \mathrm{W}): \mathrm{x} \in \mathrm{F}\right\}$ covers $\mathrm{X}$;

(4) (i) $\mathscr{A}(x)$ is precompact in $E$ for each $x \in X$;

(ii) given $W \in \mathscr{W}$, there exists a finite set $F \subseteq X$ such that $\left\{G_{x}(\mathscr{A}, W): x \in F\right\}$ covers $X$.

Definition 3. A subset $P$ of $\mathbb{R}$ is called relatively dense in $\mathbb{R}$ if there exists a number $\ell>0$ such that every interval of length $\ell$ in $\mathbb{R}$ contains at least one point of $P$.

Definition 4. A function $f: \mathbb{R} \rightarrow E$ is called almost periodic if it is continuous and, for each $W \in \mathscr{W}$, there exists a number $\ell=\ell(W, f)>0$ such that each interval of length $\ell$ in $\mathbb{R}$ contains a point $\tau=\tau(W, f)$ such that

$$
f(t+\tau)-f(t) \in W \quad \forall t \in \mathbb{R} .
$$

A number $\tau \in \mathbb{R}$ for which (7) holds is called a $(W, f)$-translation number of $f$. The above property says that, for each $W \in \mathscr{W}$, the function $f$ has a set of $(W, f)$-translation numbers $P=P(W, f)$ which is relatively dense in $\mathbb{R}$. The set of all almost periodic functions $f: \mathbb{R} \rightarrow E$ is denoted by $A P(\mathbb{R}, E)$. For any $f: \mathbb{R} \rightarrow E$ and a fixed $\omega \in \mathbb{R}$, the $\omega$ translate of $f$ is defined as the function $f_{\omega}: \mathbb{R} \rightarrow E$ defined by

$$
f_{\omega}(t)=f(t+\omega), \quad t \in \mathbb{R} .
$$

We will denote $H(f)=\left\{f_{\omega}: \omega \in \mathbb{R}\right\}$, the set of all translates of $f$.

Theorem 5 (see $[4,7])$. Let $E$ be a TVS. Let $f \in A P(\mathbb{R}, E)$. Then,

(a) $f$ has totally bounded range $f(\mathbb{R})$; hence $f$ is bounded;

(b) $f$ is uniformly continuous on $\mathbb{R}$.

Remark 6. Clearly, by the above theorem, $A P(\mathbb{R}, E) \subseteq$ $C_{b}(\mathbb{R}, E)$. We shall see below that $A P(\mathbb{R}, E)$ is a vector space.

Theorem 7 (see $[4,7]$ ). Let $E$ be a TVS. If $\left\{f_{n}\right\}$ is a sequence in $A P(\mathbb{R}, E)$ such that $f_{n} \stackrel{u}{\rightarrow} f$, then $f \in A P(\mathbb{R}, E)$.

Theorem 8 (Bochner's criterion [4, 7]). Let E be a TVS and $f: \mathbb{R} \rightarrow$ E a continuous function.

(a) If the set of translates $H(f)=\left\{f_{\omega}: \omega \in \mathbb{R}\right\}$ is $u$-sequentially compact in $C_{b}(\mathbb{R}, E)$, then $f$ is almost periodic.

(b) Conversely, if $f$ is almost periodic and, in addition, $E$ is an F-space, then the set of translates $H(f)=\left\{f_{\omega}: \omega \in\right.$ $\mathbb{R}$ \} is $u$-compact in $C_{b}(\mathbb{R}, E)$.

Thus, for $E$ being an F-space, a continuous function $f$ : $\mathbb{R} \rightarrow E$ is almost periodic if and only if the set $H(f)=\left\{f_{\omega}\right.$ : $\omega \in \mathbb{R}\}$ is $u$-compact in $C_{b}(\mathbb{R}, E)$.

Theorem 9 (see $[4,7]$ ). Let $E$ be a TVS. If $f: \mathbb{R} \rightarrow E$ is almost periodic, then the functions (i) $\lambda f(\lambda \in \mathbb{K})$, (ii) $\bar{f}(t) \equiv$ $f(-t)$, and (iii) $f_{\omega}(t)=f(t+\omega)(\omega \in \mathbb{R})$ are also almost periodic. 
Theorem 10 (see[4, 7]). Let $E$ be an F-space.

(a) If $f, g \in A P(\mathbb{R}, E)$, then $f+g \in A P(\mathbb{R}, E)$; hence $A P(\mathbb{R}, E)$ is a vector space.

(b) $(A P(\mathbb{R}, E), u)$ is complete.

\section{Main Results}

For a fixed $a \in \mathbb{R}$, let $J_{a}=[0, \infty)=\{t \in \mathbb{R}: t \geqq a\}$. A subset $P$ of $J_{a}$ is said to be relatively dense in $J_{a}$ if there exists $\ell>0$ such that, for each $t \in J_{a}$, the closed interval $[t, t+\ell]$ contains at least one member of $P$.

We define the notion of asymptotic almost periodicity in the case where the range is in a general topological vector space $E$ as follows.

Definition 11. Consider a fixed $a \in \mathbb{R}$. A continuous function $f: J_{a} \rightarrow E$ will be called asymptotically almost periodic if, given any $W \in \mathscr{W}$, there exists $r=r(W, f) \geqq a$ and a relatively dense set $P=P(W, f)$ in $J_{r}$ such that, for each $\tau \in P$ and every $t \in J_{r}$ with $t+\tau \geqq r$,

$$
f(t+\tau)-f(t) \in W .
$$

In this section, we obtain extension of some results of [13] to our general setting.

Definition 12. Consider a fixed $a \in \mathbb{R}$. A subset $\mathscr{A}$ of $C\left(J_{a}, E\right)$ is called

(i) equialmost periodic if, given $W \in \mathscr{W}$, there exists a relatively dense set $P=P(W, \mathscr{A})$ in $\mathbb{R}$ such that, for each $\tau \in P$ and every $t \in \mathbb{R}$,

$$
f(t+\tau)-f(t) \in W \quad \forall f \in \mathscr{A} ;
$$

(ii) equiasymptotically almost periodic if, given $W \in \mathscr{W}$, there exists $r=r(W, \mathscr{A}) \geqq a$ and a relatively dense set $P=P(W, \mathscr{A})$ in $J_{r}$ such that, for each $\tau \in P$ and every $t \in J_{r}$ with $t+\tau \geqq r$,

$$
f(t+\tau)-f(t) \in W \quad \forall f \in \mathscr{A} .
$$

Lemma 13. (a) For any $a \in \mathbb{R}$, let $T=J_{a}$, and let $\mathscr{A}$ be a precompact subset of $(C(T, E), k)$. If $\mathscr{A}$ is also equi-asymptotically almost periodic, then $\mathscr{A}$ is uniformly equicontinuous on $T$ and $\mathscr{A}(T)$ is precompact in $E$.

(b) Let $T=\mathbb{R}$, and let $\mathscr{A}$ be a precompact subset of $(C(T, E), k)$. If $\mathscr{A}$ is equi-almost periodic, then $\mathscr{A}$ is uniformly equicontinuous on $T$ and $\mathscr{A}(T)$ is precompact in $E$.

Proof. (a) Suppose $T=J_{a}$ with $\mathscr{A}$ being a precompact subset of $(C(T, E), k)$ and also equi-asymptotically almost periodic.

First, $\mathscr{A}$ is uniformly equicontinuous on $J_{a}$ as follows. Let $W \in \mathscr{W}$. Choose balanced $V \in \mathscr{W}$ such that $V+V+V \subseteq W$. There exist $r \geqq a, \ell>0$, and a relatively dense set $P$ in $J_{r}$ such that, for each $\tau \in P$ and every $t \in J_{r}$ with $t+\tau \geqq r$,

$$
f(t+\tau)-f(t) \in V \quad \forall f \in \mathscr{A},
$$

while $[t, t+\ell] \cap P \neq \varnothing$ for any $t \in J_{r}$. Let $d=\max \{r, \ell\}$, and choose $\tau_{k} \in[k d,(k+1) d] \cap P, k=1,2, \ldots$ By Theorem 1 , $\mathscr{A}$ is equicontinuous on $J_{a}$, and hence $\mathscr{A}$ is uniformly equicontinuous on the closed interval $[a, 5 d]$. Then there exists $\delta \in(0, d / 2)$ such that

$$
\begin{gathered}
f\left(t_{1}\right)-f\left(t_{2}\right) \in V \text { whenever } f \in \mathscr{A}, \\
t_{1}, t_{2} \in[a, 5 d] \text { with }\left|t_{1}-t_{2}\right|<\delta .
\end{gathered}
$$

Now, let $t_{1}, t_{2}>4 d$ with $\left|t_{1}-t_{2}\right|<\delta$. Choose $k \in \mathbb{N}$ such that $t_{1}, t_{2} \in[k d,(k+2) d]$ and put $s_{1}=t_{1}-\tau_{k-2}, s_{2}=t_{2}-\tau_{k-2}$. Then it is easy to see that $s_{1}, s_{2} \in[d, 4 d]$ and $\left|s_{1}-s_{2}\right|<\delta$, and so by (12) and (13),

$$
\begin{aligned}
f\left(t_{1}\right)-f\left(t_{2}\right)= & \left(f\left(s_{1}+\tau_{k-2}\right)-f\left(s_{1}\right)\right) \\
& +\left(f\left(s_{1}\right)-f\left(s_{2}\right)\right) \\
& +\left(f\left(s_{2}+\tau_{k-2}\right)-f\left(s_{2}\right)\right) \\
& \in V+V+V \subseteq W
\end{aligned}
$$

for any $f \in \mathscr{A}$. This shows that $\mathscr{A}$ is uniformly equicontinuous on $J_{a}$.

Next, $\mathscr{A}\left(J_{a}\right)$ is precompact in $E$ as follows. Let $W, V, r, \ell, P$, and $d$ be as above. By the equicontinuity of $\mathscr{A}$, we can choose a finite (open) cover $\left\{G_{i}: 1 \leq i \leq n\right\}$ of $[a, 3 d]$ and $t_{i} \in G_{i}, i=$ $1, \ldots, n$, such that

$$
\begin{gathered}
f(t)-f\left(t_{i}\right) \in V \text { for } t \in G_{i}, \\
i \in\{1, \ldots, n\} \text { and all } f \in \mathscr{A} .
\end{gathered}
$$

If $t>3 d$, choose $k \in \mathbb{N}$ such that $t \in[k d,(k+1) d]$. Putting $s=t-t_{k-2}$, we have $s \in[d, 3 d]$. Then $s \in G_{i}$ for some $i \in$ $\{1, \ldots, n\}$, and therefore, for any $f \in \mathscr{A}$, by (12) and (15),

$$
\begin{array}{r}
f(t)-f\left(t_{i}\right) \\
=\left(f\left(s+\tau_{k-2}\right)-f(s)\right)+\left(f(s)-f\left(t_{i}\right)\right) \\
\quad \in V+V \subseteq W .
\end{array}
$$

That is, $\mathscr{A}\left(J_{a}\right) \subseteq \bigcup_{i=1}^{n}\left(\mathscr{A}\left(t_{i}\right)+W\right)$. By Theorem 1 , for each $i \in$ $\{1, \ldots, n\}, \mathscr{A}\left(t_{i}\right)$ is precompact in $E$. Thus $\mathscr{A}\left(J_{a}\right)$ is precompact in $E$.

(b) Suppose $T=\mathbb{R}$, and let $\mathscr{A}$ be a precompact subset of $(C(T, E), k)$ and also equi-almost periodic. Then, with minor changes in the above proof, it follows that $\mathscr{A}$ is uniformly equicontinuous on $T$ and $\mathscr{A}(T)$ is precompact in $E$.

Theorem 14. Let $E$ be a TVS, and let $T=J_{a}$, where $a \in \mathbb{R}$. Then the following are equivalent for a subset $\mathscr{A}$ of $C(T, E)$.

(1) Consider the following:

(i) $\mathscr{A}$ is precompact in $(C(T, E), k)$;

(ii) $\mathscr{A}$ is equi-asymptotically almost periodic.

(2) The set $H^{+}(\mathscr{A})=\left\{f_{\omega}: f \in \mathscr{A}, \omega \in \mathbb{R}^{+}\right\}$of translates is a precompact subset of $\left(C_{b}(T, E), u\right)$. 
Proof. (1) $\Rightarrow$ (2) First, we consider the case $T=J_{a}$ and assume that $\mathscr{A}$ is a $k$-precompact and equi-asymptotically almost periodic subset of $C\left(J_{a}, E\right)$. By Lemma $13, \mathscr{A}\left(J_{a}\right)$ is precompact in $E$. Since $H^{+}(\mathscr{A})$ is contained in $\left(C_{p c}\left(J_{a}, E\right), u\right)$, $H^{+}(\mathscr{A})(t)$ is precompact in $E$ for each $t \in J_{a}$. To show that $H^{+}(\mathscr{A})$ is precompact in $\left(C_{p c}\left(J_{a}, E\right), u\right)$, we need to verify that the finite covering condition 3(ii) of Arzelà-Ascoli Theorem 2 holds for $H^{+}(\mathscr{A})$ in this setting. [Let $W \in \mathscr{W}$; choose a balanced $V \in \mathscr{W}$ such that $V+V+V \subseteq W$. As in Lemma 13, choose $r \geqq a, l>0$, and a relatively dense set $P$ in $J_{r}$ such that, for each $\tau \in P$ and every $t \in J_{r}$ with $t+\tau \geqq r$,

$$
f(t+\tau)-f(t) \in V \quad \forall f \in \mathscr{A},
$$

while $[t, t+l] \cap P \neq \varnothing$ for any $t \in J_{r}$. Furthermore, we put $d=\max \{r, l\}$, set $\tau_{0}=0$, and fix $\tau_{k} \in[k d,(k+1) d] \cap$ $P, k=1,2, \ldots$. By Lemma $13, \mathscr{A}$ is uniformly equicontinuous on $J_{a} ; H^{+}(\mathscr{A})$ is also clearly uniformly equicontinuous. In particular, we obtain a finite cover $\left\{S_{i}: 1 \leq i \leq n\right\}$ of $[d, 3 d]$ by (relatively open) subsets of $J_{r}$ and $s_{i} \in S_{i}, i=1, \ldots, n$, such that, for every $f \in \mathscr{A}$ and all $\omega \in \mathbb{R}^{+}$,

$$
\begin{aligned}
& f_{\omega}(s)-f_{\omega}\left(s_{i}\right) \in V \\
& \quad \text { whenever } s \in S_{i}, \quad i \in\{1, \ldots, n\} .
\end{aligned}
$$

Put $G_{i}=\bigcup_{k=0}^{\infty}\left(S_{i}+\tau_{k}\right), i=1, \ldots, n$. Now, taking $t \in G_{i}$ for $i \in\{1, \ldots, n\}$, choose $s \in S_{i}$ and $k \in \mathbb{N} \cup\{0\}$ so that $t=s+\tau_{k}$. Using (17) and (18), we obtain

$$
\begin{aligned}
f_{\omega}(t)-f_{\omega}\left(s_{i}\right) & \\
=\left(f\left(s+\omega+\tau_{k}\right)-f(s+\omega)\right)+( & \left.f_{\omega}(s)-f_{\omega}\left(s_{i}\right)\right) \\
& \in V+V \subseteq W
\end{aligned}
$$

for every $f \in \mathscr{A}$ and all $\omega \in \mathbb{R}^{+}$.

Next, for any $\mathscr{A} \subseteq C_{p c}\left(J_{a}, E\right)$ and $W \in \mathscr{W}$, recall the notation (6):

$$
\begin{array}{r}
G_{s}(\mathscr{A}, W) \\
=\left\{t \in J_{a}: f(t)-f(s) \in W \quad \forall f \in \mathscr{A}\right\}, \\
s \in J_{a} .
\end{array}
$$

It is easy to verify that $J_{d}=[d, \infty) \subseteq \bigcup_{i=1}^{n} G_{i}$, and so $\left\{G_{s_{i}}\left(H^{+}(\mathscr{A}), W\right): i=1, \ldots, n\right\}$ covers $J_{d}$. By the equicontinuity of $H^{+}(\mathscr{A})$, it is possible to trivially cover $[a, d]$ by finitely many sets of this same prescribed form, we see that 3(ii) of Arzelà-Ascoli Theorem 2 is satisfied. Thus $H^{+}(\mathscr{A})$ is precompact in $\left(C_{b}\left(J_{a}, E\right), u\right)$.

(2) $\Rightarrow$ (1) Assume that (6) holds.

(1)(i) This is clearly satisfied.

(1)(ii) In view of the Arzelà-Ascoli Theorem $1, H^{+}(\mathscr{A})$ is a precompact subset of $\left(C_{b}\left(J_{a}, E\right), u\right)$. Furthermore, the ArzelàAscoli Theorem 2 can be used in showing that $H^{+}(\mathscr{A})$ is equiasymptotically almost periodic as follows. Fixing $W \in \mathscr{W}$, we use (3)(ii) of Arzelà-Ascoli Theorem 2 to obtain a finite cover $\left\{G_{i}: 1 \leq i \leq n\right\}$ of $J_{b}$, where $b=\max \{a, 1\}$ and $G_{i} \subseteq J_{b}$, $i=1, \ldots, n$, and $t_{i} \in G_{i}, i=1, \ldots, n$, such that, for every $f \in \mathscr{A}$ and all $\omega \in \mathbb{R}^{+}$,

$$
f_{\omega}(t)-f_{\omega}\left(t_{i}\right) \in W \quad \text { whenever } t \in G_{i}, i \in\{1, \ldots, n\} .
$$

Putting $r=\ell=\max \left\{t_{1}, \ldots, t_{n}\right\}$, we note that $r \geqq a$ and $\ell>0$. Now, let $P=\left(\bigcup_{i=1}^{n}\left(G_{i}-t_{i}\right)\right) \cap J_{r}$. Then $P$ is relatively dense in $J_{r}$. (For any $t \in J_{r}, t+\ell \geqq b$, we have $t+\ell \in G_{i}$ for some $i \in\{1, \ldots, n\}$. Then $t \leqq(t+\ell)-t_{i} \leqq t+\ell$.) Now, given $t \in J_{r}$ and $\tau \in P$, choose $i \in\{1, \ldots, n\}$ and $s \in G_{i}$ such that $\tau=s-t_{i}$. Since $t-t_{i} \geqq 0$, we then have by (21)

$$
\begin{aligned}
f( & +\tau)-f(t) \\
& =f\left(\left(t-t_{i}\right)+s\right)-f\left(\left(t-t_{i}\right)+t_{i}\right) \\
& =f_{t-t_{i}}(s)-f_{t-t_{i}}\left(t_{i}\right) \in W
\end{aligned}
$$

for all $f \in \mathscr{A}$. This proves that $\mathscr{A}$ is equi-asymptotically almost periodic.

We next obtain an analogue of the above result for $T=\mathbb{R}$ (instead of $T=J_{a}$ ) and $\mathscr{A}$ equi-almost periodic (instead of equi-asymptotically almost periodic) as follows.

Theorem 15. Let $E$ be a TVS, and let $T=\mathbb{R}$. Then the following are equivalent for a subset $\mathscr{A}$ of $C(T, E)$.

(1) (i) $\mathscr{A}$ is precompact in $(C(T, E), k)$;

(ii) $\mathscr{A}$ is equi-almost periodic.

(2) The set $H(\mathscr{A})=\left\{f_{\omega}: f \in \mathscr{A}, \omega \in \mathbb{R}\right\}$ of translates is a precompact subset of $\left(C_{b}(T, E), u\right)$.

Proof. This follows from Theorem 14 with minor changes, outlined as follows.

$(1) \Rightarrow$ (2) Suppose $\mathscr{A}$ is a $k$-precompact and equialmost periodic subset of $C(\mathbb{R}, E)$. For this part, we can easily adapt the arguments of Theorem 14 to show that $H(\mathscr{A})$ is a precompact subset of $\left(C_{p c}(\mathbb{R}, E), u\right)$.

$(2) \Rightarrow$ (1) In view of the Arzelà-Ascoli Theorem $1, \mathscr{A}$ is a precompact subset of $\left(C_{p c}(\mathbb{R}, E), u\right)$. Further, $\mathscr{A}$ is equialmost periodic. In fact, fix $W \in \mathscr{W}$. Since $H(\mathscr{A})$ is a precompact, we use (3)(ii) of Arzelà-Ascoli Theorem 2 to obtain a finite cover $\left\{G_{i}: 1 \leq i \leq n\right\}$ of $\mathbb{R}$ and $t_{i} \in G_{i}, i=$ $1, \ldots, n$, such that, for every $f \in \mathscr{A}$ and all $\omega \in \mathbb{R}$,

$$
f_{\omega}(t)-f_{\omega}\left(t_{i}\right) \in W \quad \text { whenever } t \in G_{i}, i \in\{1, \ldots, n\} .
$$

In this case, take $\ell>2 \max \left\{\left|t_{1}\right|, \ldots,\left|t_{n}\right|\right\}$, and put $P=$ $\bigcup_{i=1}^{n}\left(G_{i}-t_{i}\right)$. Then $P$ is relatively dense in $\mathbb{R}$. In fact, for any $t \in \mathbb{R}$, choose $i \in\{1, \ldots, n\}$ such that $t+\ell / 2 \in G_{i}$. Then

$$
\left(t+\frac{\ell}{2}\right)-t_{i} \in[t, t+\ell] \cap P .
$$

Also, given $t \in \mathbb{R}$ and any $\tau \in P$, we can adapt the arguments of Theorem 14 till proving (22) and obtain

$$
f(t+\tau)-f(t) \in W \quad \forall f \in \mathscr{A} .
$$

Consequently, $\mathscr{A}$ is equi-almost periodic. 
From Theorem 14, we can deduce the following extension of Fréchet's theorem.

Theorem 16. Let $E$ be a TVS, and let $a \in \mathbb{R}$. For any $f \in$ $C\left(J_{a}, E\right), f$ is asymptotically almost periodic if and only if the set $H^{+}(f)=\left\{f_{\omega}: \omega \geqq 0\right\}$ is a precompact subset of $\left(C_{b}\left(J_{a}, E\right), u\right)$.

We next consider an alternate view of asymptotically almost periodic functions. Fixing $a \in \mathbb{R}$, if $g: \mathbb{R} \rightarrow E$ is a continuous almost periodic function and $f \in C_{0}\left(J_{a}, E\right)$, then the function $f=g \mid J_{a}+h$ is clearly asymptotically almost periodic on the interval $J_{a}$. On the other hand, it is also known that every asymptotically almost periodic function in $C\left(J_{a}, E\right)$ can be so represented in case $E$ is finite dimensional. We shall use Theorem 14 to study it in a more general situation. Recall that a TVS $E$ is said to be quasicomplete if every bounded Cauchy net in $E$ converges. Clearly completeness implies quasicompleteness.

Theorem 17. Assume that $E$ is a quasi-complete TVS, and fix $a \in \mathbb{R}$. Then $f \in C\left(J_{a}, E\right)$ is asymptotically almost periodic if and only if there is a unique almost periodic function $g \in$ $C(\mathbb{R}, E)$ and a unique function $h \in C_{0}\left(J_{a}, E\right)$ such that

$$
f=g \mid J_{a}+h \text {. }
$$

Proof. Since $E$ is a quasi-complete TVS, it follows that both $\left(C_{p c}\left(J_{a}, E\right), u\right)$ and $\left(C_{p c}(\mathbb{R}, E), u\right)$ are quasi-complete $[13,16$, 18]. Therefore, precompactness can be considered equivalent to relative compactness in either of these two spaces.

Consider an arbitrary asymptotically almost periodic function $f \in C\left(J_{a}, E\right)$. Then, for each pair $(n, W) \in \mathbb{N} \times \mathscr{W}$, there exist $r(n, W) \geqq \max \{a, n\}$ and a relatively dense set $P(n, W)$ in $J_{r(n, W)}$ such that, for any $\tau \in P(n, W)$ and every $t \in J_{r(n, W)}$,

$$
f(t+\tau)-f(t) \in \frac{1}{n} W .
$$

Let us equip $D=\mathbb{N} \times \mathscr{W}$ with the usual product order; that is, given $(m, V),(n, W) \in D,(m, V) \leqq(n, W)$ if and only if $m \leqq n$ and $W \subseteq V$ (or equivalently $\rho_{V} \subseteq \rho_{W}$ ). Also, for each $\alpha=(n, W) \in D$, we choose $\tau_{\alpha} \in P(n, W)$. By Theorem 14, since $\left\{\tau_{\alpha}\right\}_{\alpha \in D}$ is a net in $\mathbb{R}^{+}$, there is a subnet $\left\{\tau_{\alpha(\lambda)}\right\}_{\lambda \in D}$ of $\left\{\tau_{\alpha}\right\}_{\alpha}$ for which the net of translates $\left\{f_{\tau_{\alpha(\lambda)}}\right\}$ converges uniformly on $J_{a}$ to some $\varphi \in\left(C_{p c}\left(J_{a}, E\right), u\right)$.

We claim that $\varphi$ has an almost periodic extension $g \in$ $C(\mathbb{R}, E)$. For a fixed $b \in \mathbb{R} \backslash J_{a}=(-\infty, a)$, there exists $\lambda_{b} \in D$ so that $b+\tau_{\alpha(\lambda)} \geqq a$ whenever $\lambda \in D$ and $\lambda \geqq \lambda_{b}$; put $D_{b}=\left\{\lambda \in D: \lambda \geqq \lambda_{b}\right\}$. For each $\lambda \in D_{b}$, let $f_{\tau_{\alpha(\lambda)}}^{*}: J_{b} \rightarrow X$ be an extension of $f_{\tau_{\alpha(\lambda)}}$ defined by

$$
f_{\tau_{\alpha(\lambda)}}^{*}(s)=f\left(s+\tau_{\alpha(\lambda)}\right), \quad s \in J_{b} .
$$

We can easily see that $\left\{f_{\tau_{\alpha(\lambda)}^{*}}^{*}: \lambda \in D_{b}\right\}$ is a bounded net in $\left(C_{p c}\left(J_{a}, E\right), u\right)$. Now, let $\varepsilon>0$ and $W \in \mathscr{W}$ be given. Choose $V \in \mathscr{W}$ with $V+V+V \subseteq W$ and choose $n \in \mathbb{N}$ with $n \geqq$ $\max \{a-b, 3 / \varepsilon\}$, let $\tau \in P(n, V)$, and take $\lambda_{0} \in D_{b}$ for which the following conditions are satisfied. (i) If $\lambda, \mu \in D_{b}$ and $\lambda, \mu \geqq \lambda_{0}$, then

$$
\begin{gathered}
f_{\tau_{\alpha(\lambda)}}(t)-f_{\tau_{\alpha(\mu)}}(t) \in \frac{\varepsilon}{3} V \quad \forall t \in J_{a}, \\
\text { or } \rho_{V}\left[f_{\tau_{\alpha(\lambda)}}(t)-f_{\tau_{\alpha(\mu)}}(t)\right] \leq \frac{\varepsilon}{3} \quad \forall t \in J_{a} .
\end{gathered}
$$

(ii) In case $\lambda \in D_{b}$ and $\lambda \geqq \lambda_{0}, b+\tau_{\alpha(\lambda)} \geqq r(n, V)$. For any $s \in J_{b}$, if $\lambda, \mu \geqq \lambda_{0}$ (as in case (i)), we then have by (2), (28), and (29) that

$$
\begin{aligned}
\rho_{W}[ & \left.f_{\tau_{\alpha(\lambda)}}^{*}(s)-f_{\tau_{\alpha(\lambda)}}^{*}(s)\right] \\
\leq & \rho_{V}\left(f\left(s+\tau_{\alpha(\lambda)}\right)-f\left(s+\tau_{\alpha(\lambda)}+\tau\right)\right) \\
& +\rho_{V}\left(f_{\tau_{\alpha(\lambda)}}(s+\tau)-f_{\tau_{\alpha(\mu)}}(s+\tau)\right) \\
& +\rho_{V}\left(f\left(s+\tau_{\alpha(\mu)}+\tau\right)-f\left(s+\tau_{\alpha(\mu)}\right)\right) \\
\leq & \frac{\varepsilon}{3}+\frac{\varepsilon}{3}+\frac{\varepsilon}{3}=\varepsilon .
\end{aligned}
$$

So $\left\{f_{\tau_{\alpha(\lambda)}}\right\}_{\lambda \in D_{b}}$ is a Cauchy net in $\left(C_{p c}\left(J_{a}, E\right), u\right)$. Since it is also bounded and $\left(C_{p c}\left(J_{a}, E\right), u\right)$ is quasi-complete, it converges uniformly on $J_{b}$ to a function $\varphi_{b} \in\left(C_{p c}\left(J_{a}, E\right), u\right)$. Clearly, we have that $\varphi_{b} \mid J_{a}=\varphi$. If $c \in \mathbb{R}$ with $c \leqq b$ and if $\lambda_{c}$ is any element in $D$ for which $c+\tau_{\alpha(\lambda)} \geqq a$ for all $\lambda \in D$ such that $\lambda \geqq$ $\lambda_{0}$, then the corresponding net $\left\{f_{\tau_{\alpha(\lambda)}}\right\}_{\lambda \geqq \lambda_{c}}$ of extensions from $J_{a}$ to $J_{c}$ will converge in $C_{p c}\left(J_{c}, E\right)$ to a function $\varphi_{c}$. Clearly $\varphi_{c}=\varphi_{b}$ on $J_{b}$. Define a function $g: \mathbb{R} \rightarrow E$ by

$$
g(t)= \begin{cases}\varphi(t), & t \in J_{a} \\ \varphi_{t}(t), & t \in \mathbb{R} \backslash J_{a} .\end{cases}
$$

Then $g$ is well defined and continuous on $\mathbb{R}$ and $g \mid J_{a}=\varphi$.

We claim that $g$ is also almost periodic. Let $\varepsilon>0$ and $W \in \mathscr{W}$. Choose a balanced $U \in \mathscr{W}$ with $U+U+U \subseteq W$. Choose $n \in \mathbb{N}$ such that $n \geqq 3 / \varepsilon$ and the set $P=P(n, W) \cup\{\tau \in$ $\mathbb{R}:-\tau \in P(n, W)\}$ is relatively dense in $\mathbb{R}$. Next, given $t \in \mathbb{R}$ and $\tau \in P$, first choose $b \in \mathbb{R} \backslash J_{a}$ with $b \leqq \min \{t, t+\tau\}$, and then take $\lambda \in D$ such that

(a) $b+\tau_{\alpha(\lambda)} \geqq r(n, W)+|\tau|$,

(b) $\varphi_{b}(s)-f\left(s+\tau_{\alpha(\lambda)}\right) \in(\varepsilon / 3) U$ for every $s \in J_{b}$.

Then, by (2), (31), and (b),

$$
\begin{aligned}
\rho_{W}[ & g(t+\tau)-g(t)] \\
\leq & \rho_{U}\left(\varphi_{b}(t+\tau)-f\left(t+\tau+\tau_{\alpha(\lambda)}\right)\right) \\
& +\rho_{U}\left(f\left(\tau+\tau_{\alpha(\lambda)}+\tau\right)-f\left(\tau+\tau_{\alpha(\lambda)}\right)\right) \\
& +\rho_{U}\left(f\left(\tau+\tau_{\alpha(\lambda)}\right)-\varphi_{b}(t)\right) \\
\leq & \frac{\varepsilon}{3}+\frac{\varepsilon}{3}+\frac{\varepsilon}{3}=\varepsilon,
\end{aligned}
$$

and so $g$ is almost periodic.

It remains to show that $h=f-g \mid J_{a}$ vanishes at infinity on $J_{a}$. Fix $W \in \mathscr{W}$. Choose a balanced $V \in \mathscr{W}$ with $V+V \subseteq$ 
$W$, and choose sufficiently large $n \in \mathbb{N}$ such that there exists $\lambda \in D$ such that $\alpha(\lambda) \geqq(n, V)$ and

$$
f_{t_{\alpha}(\lambda)}(t)-\varphi(t) \in V \quad \forall t \in J_{a}
$$

we may assume that $\alpha(\lambda)=(m, V)$, where $m \in \mathbb{N}$. Thus, if $t \in J_{r(m, V)}$, then

$$
\begin{aligned}
h(t)= & f(t)-\varphi(t) \\
= & \left(f(t)-f\left(t+\tau_{\alpha(\lambda)}\right)\right) \\
& +\left(f\left(t+\tau_{\alpha(\lambda)}\right)-\varphi(t)\right) \\
& \in V+V \subseteq W .
\end{aligned}
$$

Finally, we show that the functions $g$ and $h$ in the representation (26) are unique. First observe that an almost periodic function $\theta \in C(\mathbb{R}, E)$ must be identically zero on $\mathbb{R}$ if $\theta \mid J_{a} \in C_{0}\left(J_{a}, E\right)$. Therefore, given almost periodic functions $g$ and $\varphi$ in $C(\mathbb{R}, E)$, we only need to verify that $g+\varphi$ is also almost periodic. For this, choosing any net $\left\{\omega_{\alpha}\right\}_{\alpha}$ in $\mathbb{R}$, we apply Theorem 15 (twice) to obtain a subnet $\left\{\omega_{\alpha(\lambda)}\right\}_{\lambda}$ such that the corresponding nets of translates $\left\{g_{\omega_{\alpha(\lambda)}}\right\}$ both converge in $\left(C_{p c}(\mathbb{R}, E), u\right)$. Another application of Theorem 15 now gives us that $g+\varphi$ is almost periodic.

Scope of Applications. (1) The importance of such a work has been highlighted in ([12]; [13], p. 19-20). If, for a given Banach space $E$, a linear operator $A: \mathscr{D}(A) \subseteq E \rightarrow E$ is the infinitesimal generator of a $C_{0}$-semigroup $(T(t))_{t>0}$ of bounded linear operators from $E$ to $E$, then, for any $x_{0} \in$ $\mathscr{D}(A)$, the unique strong solution of the abstract Cauchy problem

$$
\begin{gathered}
\frac{d x(t)}{d t}=A[x(t)], \quad t>0, \\
x(0)=x_{0} \in E,
\end{gathered}
$$

associated with $A$, is given by the motion $x(\cdot)=T(\cdot) x_{0}$ of $(T(t))_{t>0}$ through $x_{0}$. See also [10].

In the qualitative study of the solution, one of the problems is to determine its asymptotic behaviour as $t \rightarrow \infty$. In this regard, a useful concept is the so-called positive $\omega$ limit set

$$
\begin{aligned}
& \omega^{+}\left(x_{0}\right) \\
& =\left\{y \in E: \exists 0 \leq t_{n} \longrightarrow \infty \text { such that } T\left(t_{n}\right) x_{0 .} \longrightarrow y\right\}
\end{aligned}
$$

of all possible limit points. The basic result is that: if the orbit $\gamma^{+}\left(x_{0}\right)=\left\{T(t) x_{0}: t \geq 0\right\}$ of the motion is relatively compact, then $\omega^{+}\left(x_{0}\right)$ is nonempty, compact, connected, and invariant. A qualitative much stronger mode of asymptotic behaviour results if not only $\gamma^{+}\left(x_{0}\right)$ is relatively compact, but also the set $H^{+}\left(T(\cdot) x_{0}\right)=\left\{T_{\omega}(\cdot) x_{0}: \omega>0\right\}, T_{\omega}(t) x_{0}=T(t+\omega) x_{0}$, of all translates of the motion is a relatively compact subset of the space $\left(C_{b}\left(\mathbb{R}^{+}, E\right),\|\cdot\|_{\infty}\right)$. This observation raises the following problem.

(*) Characterize those $f \in C_{b}\left(\mathbb{R}^{+}, E\right)$ for which $H^{+}(f)$ is relatively compact in $\left(C_{b}\left(\mathbb{R}^{+}, E\right),\|\cdot\|_{\infty}\right)$.
Clearly, our results contain a complete solution of problem $(*)$ in the general setting.

(2) In $[3,19]$, it has been obtained that in the (nonlocally convex) $p$-Fréchet space $E, 0<p<1$, (CP) has a unique solution $x(t)=T(t)(x)$, with

$$
T(t)(x)=\lim _{n \rightarrow \infty}\left(1-\frac{t}{n} A\right)^{-1}(x),
$$

with the limit being taken in the $p$-norm of $E$.

Our results thus widen the scope of applications of asymptotic almost periodicity to the nonlocally convex setting.

\section{Acknowledgments}

This research was funded by the Deanship of Scientific Research (DSR), King Abdulaziz University, Jeddah, under Project no. 97/130/1432. The authors, therefore, acknowledge with thanks DSR technical and financial support. The authors are also grateful to the referee for several helpful comments.

\section{References}

[1] S. Bochner, "Abstrakte fastperiodische Funktionen," Acta Mathematica, vol. 61, no. 1, pp. 149-184, 1933.

[2] D. Bugajewski and G. M. N'Guérékata, "Almost periodicity in Fréchet spaces," Journal of Mathematical Analysis and Applications, vol. 299, no. 2, pp. 534-549, 2004.

[3] S. G. Gal and G. M. N'Guérékata, "Almost periodic functions with values in $p$-Fréchet spaces, $0<p<1$," Global Journal of Pure and Applied Mathematics, vol. 3, no. 1, pp. 89-103, 2007.

[4] L. A. Khan and S. M. Alsulami, "Almost periodicity in linear topological spaces-revisited," Communications in Mathematical Analysis, vol. 13, no. 1, pp. 54-63, 2012.

[5] G. M. N’Guérékata, "Almost-periodicity in linear topological spaces and applications to abstract differential equations," International Journal of Mathematics and Mathematical Sciences, vol. 7, no. 3, pp. 529-540, 1984.

[6] G. M. N’Guérékata, "Notes on almost-periodicity in topological vector spaces," International Journal of Mathematics and Mathematical Sciences, vol. 9, no. 1, pp. 201-204, 1986.

[7] G. M. N'Guerekata, Almost Automorphic and Almost Periodic Functions in Abstract Spaces, Kluwer Academic Publishers, Plenum Press, New York, NY, USA, 2001.

[8] M. Fréchet, "Les fonctions asymptotiquement presqueperiodiques continunues," Les Comptes Rendus de l'Académie des Sciences, vol. 213, pp. 520-522, 1941.

[9] M. Fréchet, "Les fonctions asymptotiquement presqueperiodiques," Revue Scientifique, vol. 79, pp. 341-354, 1941.

[10] E. Hernández M., M. L. Pelicer, and J. P. C. Dos Santos, "Asymptotically almost periodic and almost periodic solutions for a class of evolution equations," Electronic Journal of Differential Equations, vol. 2004, no. 61, pp. 1-15, 2004.

[11] W. M. Ruess and V. Q., "Asymptotically almost periodic solutions of evolution equations in Banach spaces," Journal of Differential Equations, vol. 122, no. 2, pp. 282-301, 1995.

[12] W. M. Ruess and W. H. Summers, "Asymptotic almost periodicity and motions of semigroups of operators," Linear Algebra and Its Applications, vol. 84, pp. 335-351, 1986. 
[13] W. M. Ruess and W. H. Summers, "Compactness in spaces of vector valued continuous functions and asymptotic almost periodicity," Mathematische Nachrichten, vol. 135, pp. 7-33, 1988.

[14] S. Zaidman, "Existence of asymptotically almost-periodic and of almost-automorphic solutions for some classes of abstract differential equations," Annales des Sciences Mathématiques du Québec, vol. 13, no. 1, pp. 79-88, 1989.

[15] V. Klee, "Shrinkable neighborhoods in Hausdorff linear spaces," Mathematische Annalen, vol. 141, pp. 281-285, 1960.

[16] L. A. Khan, Linear Topological Spaces of Continuous VectorValued Functions, Academic Publications, Sofia, Bulgaria, 2013.

[17] L. A. Khan and L. Oubbi, "The Arzéla-Ascoli theorem in nonlocally convex weighted spaces," Revista de la Real Academia de Ciencias, vol. 60, pp. 107-115, 2005.

[18] J. L. Kelley and I. Namioka, Linear Topological Spaces, D. Van Nostrand, New York, NY, USA, 1963.

[19] S. G. Gal and J. A. Goldstein, "Semigroups of linear operators on $p$-Fréchet spaces, $0<p<1$," Acta Mathematica Hungarica, vol. 114, no. 1-2, pp. 13-36, 2007. 


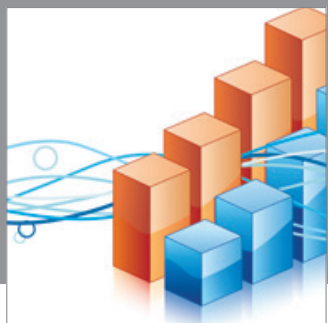

Advances in

Operations Research

mansans

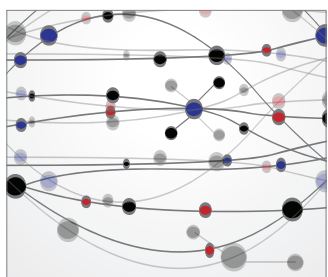

The Scientific World Journal
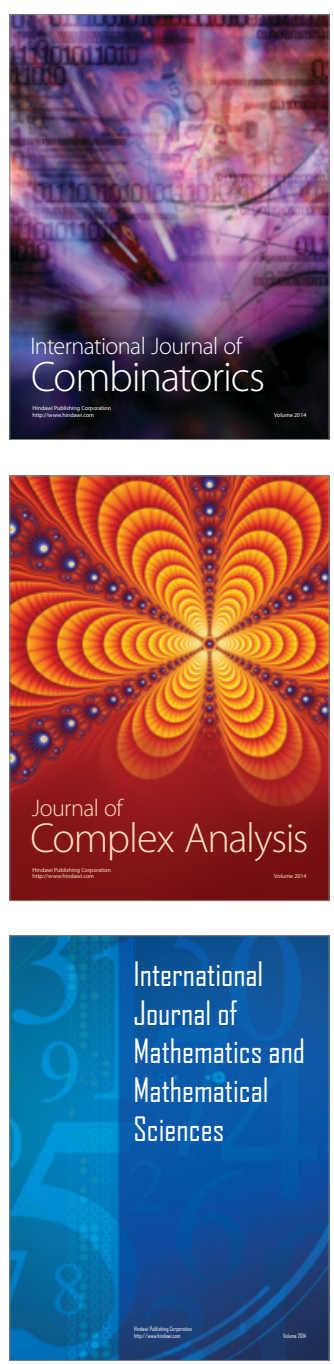
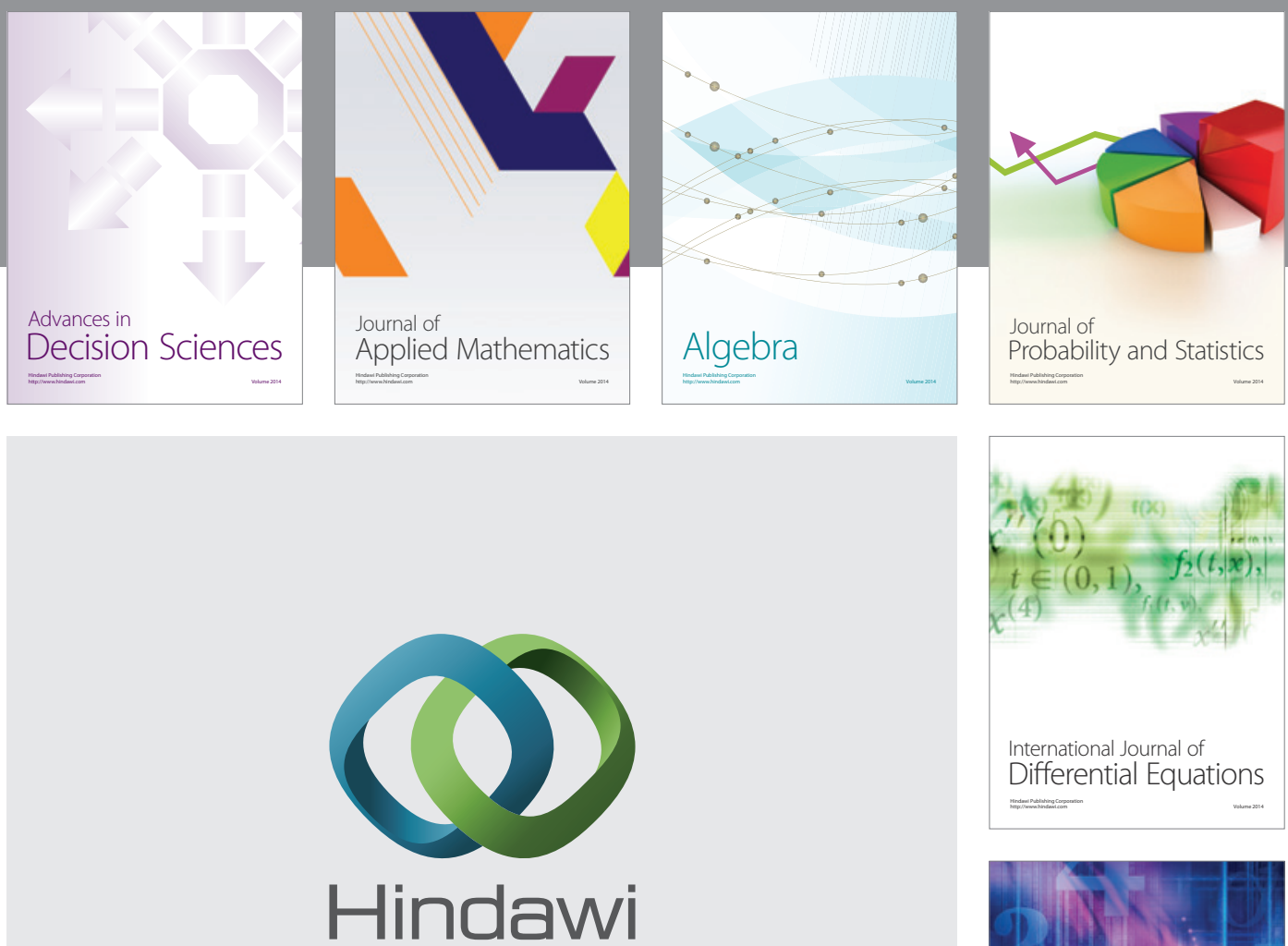

Submit your manuscripts at http://www.hindawi.com
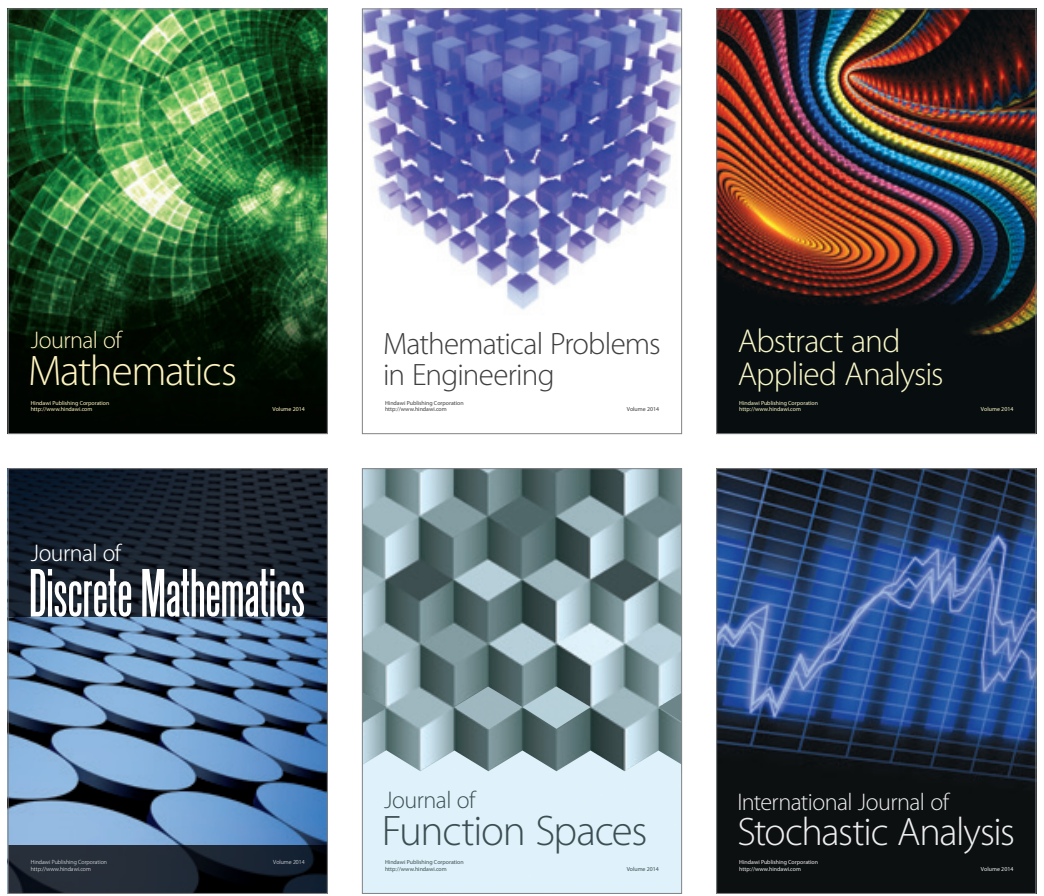

Journal of

Function Spaces

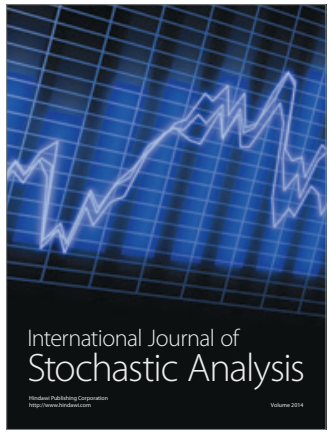

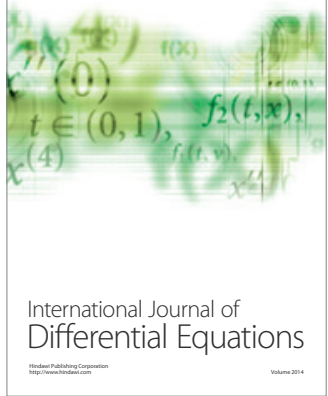
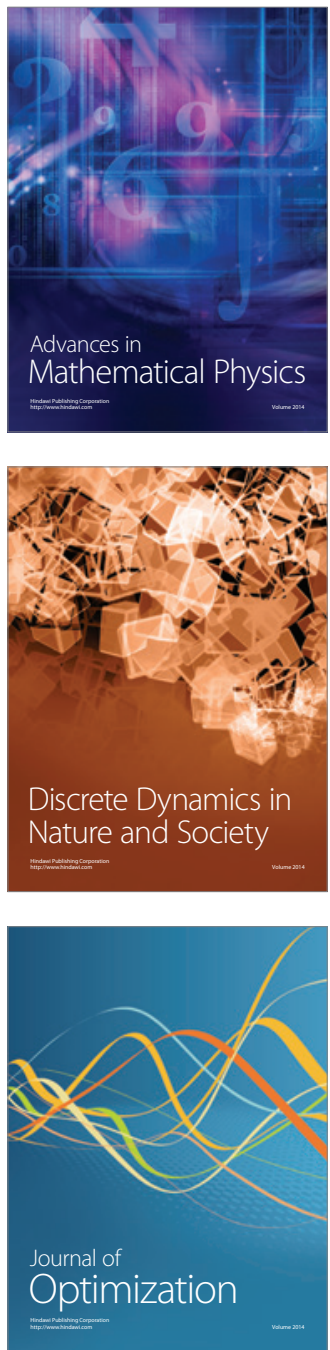